\title{
Impact and cost-effectiveness of formal gastroenterology outpatient referral Clinical Assessment Service
}

\author{
Stavroula Pelitari, ${ }^{1}$ Charlotte Hathaway, ${ }^{1}$ Dean Gritton, ${ }^{1}$ Andrea Smith, ${ }^{2}$ \\ David Bush, ${ }^{2}$ Shyam Menon, ${ }^{1}$ Brian McKaig ${ }^{1}$
}

'Department of

Gastroenterology, Royal

Wolverhampton NHS Trust,

Wolverhampton, UK

${ }^{2}$ Wolverhampton CCG,

Wolverhampton Clinical

Commissioning Group,

Wolverhampton, UK

\section{Correspondence to}

Dr Brian McKaig, Department of Gastroenterology, The Royal Wolverhampton NHS Trust, New Cross Hospital, Wolverhampton, West Midlands WV10 0QP, UK; brian.mckaig@nhs.net

Received 6 May 2017 Revised 8 August 2017

Accepted 19 August 2017

Published Online First

23 September 2017

\section{Check for updates}

To cite: Pelitari $S$,

Hathaway C, Gritton D, et al. Frontline Gastroenterology 2018;9:159-165.

\begin{abstract}
Objective The aim was to assess the financial and operational impact of our new gastroenterology referral pathway model on our services.

Design An electronic 'Clinical Assessment Service' (CAS) proforma and an information platform were developed, and all data were analysed retrospectively.

Setting Royal Wolverhampton NHS Trust. Patients 14245 general practitioner (GP) referrals were received during January 2014December 2016 with 9773 of them being triaged via our CAS.
\end{abstract}

Main outcome measures We looked into patients' clinical outcome along with departmental performance and finances. Results A new outpatient appointment was offered to $60.1 \%(n=5873)$ of the CAS referred patients. Endoscopic or radiological investigations were requested for $29.2 \%$ ( $n=2854$ ) of patients prior to deciding on further management plan. Out of those, $27 \%(n=765)$ went on to receive another gastroenterology (GI) clinic appointment. The remaining $21.3 \%(n=2089)$ of the CAS patients were discharged back to their GP following initial investigations. 5.5\% $(n=538)$ were discharged back to primary care with a letter of advice, whereas 5.2\% ( $n=509)$ were deemed inappropriate for $\mathrm{Gl}$ clinic and were redirected to other specialists. Overall, $32 \%(n=3127)$ of patients were managed without a face to face consultation in the Gl clinic. This corresponds to 3136 less outpatient appointments with estimated reduced expenditure by the Clinical Commissioning Group (CCG) of $f 481 \mathrm{~K}$. The 18week performance and waiting times remained stable despite the increasing referral population. The DNA rate dropped from $14 \%$ pre to $8.5 \%$. Conclusions Our clinical assessment model has, in addition to the clinical benefits, a considerable positive financial impact to the health economy.

\section{BACKGROUND}

In the UK, demand for specialist referrals from primary to secondary care continues to rise. This reflects a host of factors including an ageing population, health awareness campaigns, screening programmes and an increase in patients' expectations. ${ }^{12}$ In 2012-2013, gastroenterology (GI) services at Royal Wolverhampton NHS Trust (RWT) faced a $25 \%$ increase in new outpatient (OP) GI referrals compared with the preceding year. This increase in demand resulted in significant pressures, both financial and organisational, to meet national standards.

Within the GI department at RWT, it was recognised that many patients seen at a new OP appointment could potentially be managed more efficiently and reduce the number of direct face-to-face consultations required. Ensuring robust primary care work-up and adherence to agreed pathways were identified as key processes in maximising efficiency.

On this background, we collaborated with clinical colleagues from the Wolverhampton CCG (the main commissioning CCG for RWT) to discuss and develop a new model of working. The aim was to improve efficiency of our GI referral pathways and reduce total costs to the healthcare economy while delivering an improved service to our patients. Conventional referral from primary to secondary care was via three pathways; 2-week wait cancer fast track referral, choose and book or by a letter direct from general practitioner (GP) to the GI department. In an attempt to challenge this model, we developed a Clinical Assessment Service (CAS) for GP referrals to allow secondary care clinicians to triage patients to the most appropriate 
CAS Proforma

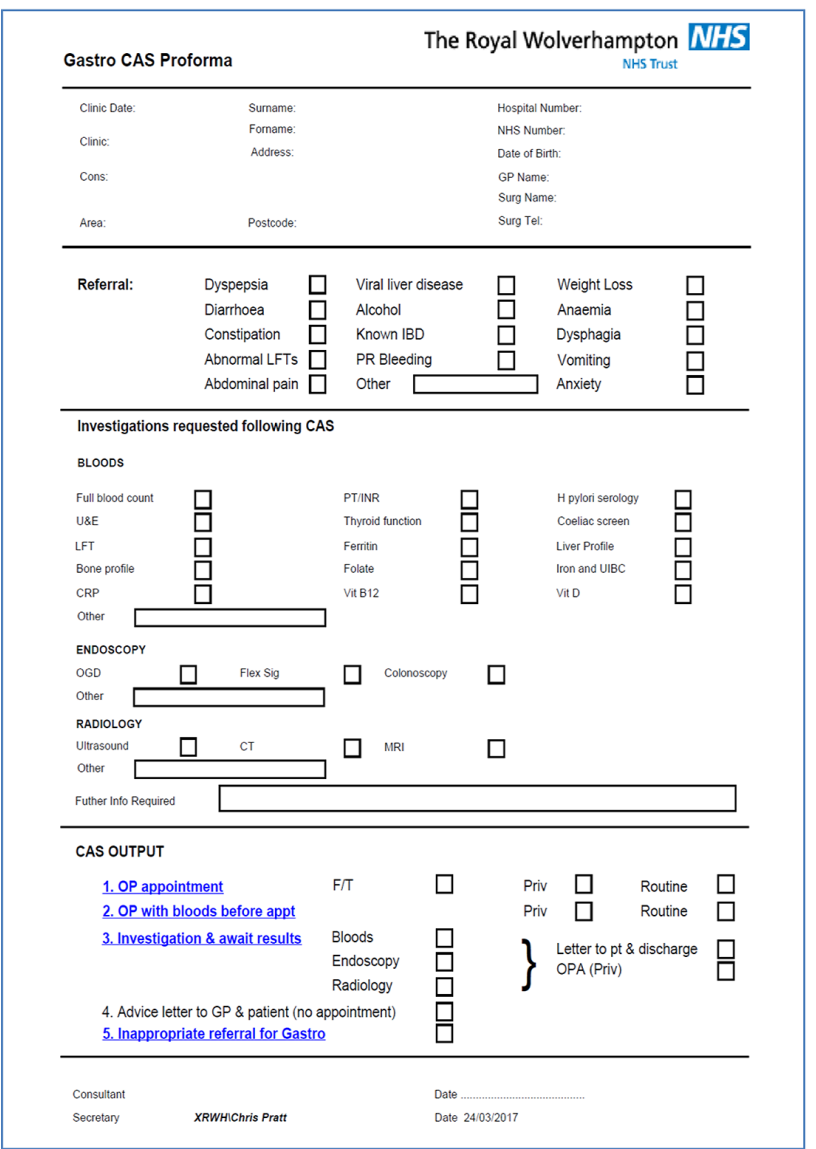

\section{CAS Flow Chart}

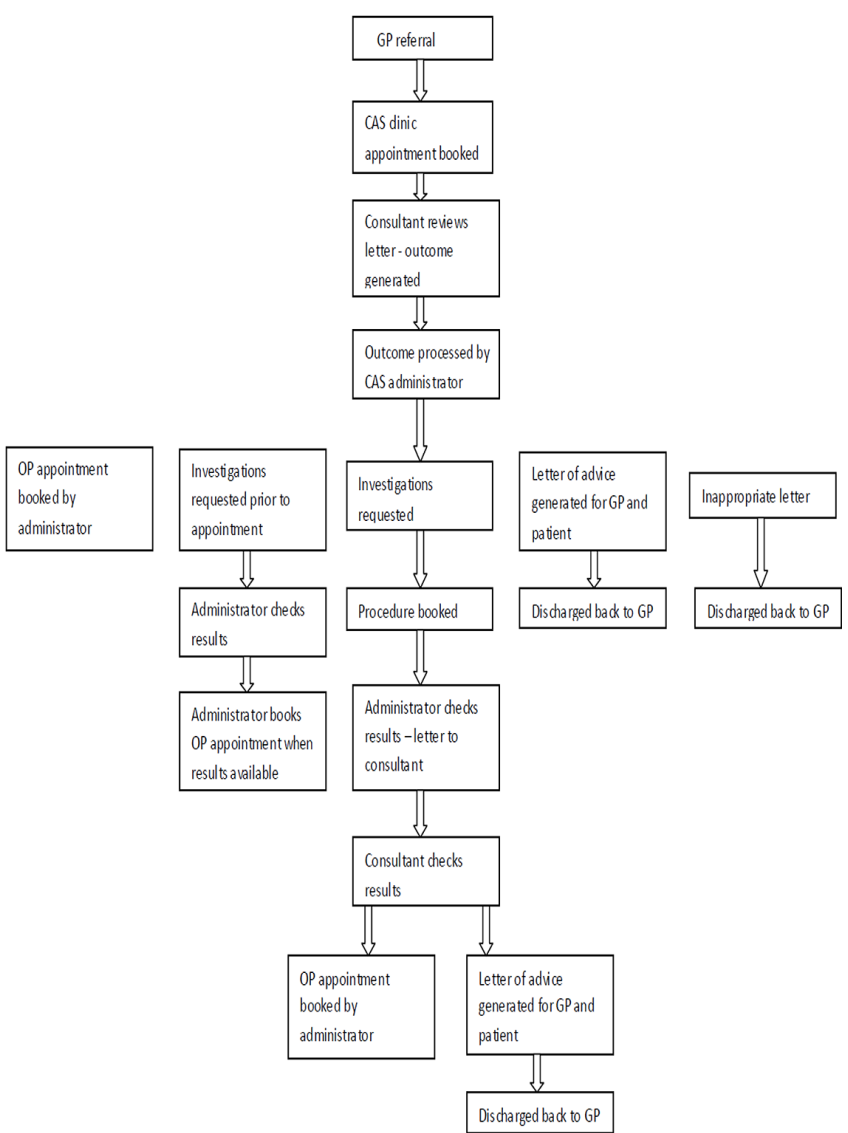

Figure 1 CAS proforma and flowchart. CAS, Clinical Assessment Service; CRP, C-reactive protein; F/T, fast track; IBD, inflammatory bowel disease; LFTs, liver function tests; OGD, oesophagogastroduodenoscopy; OPA, outpatient appointment; PR, per rectum; PT/INR, prothrombin time/ international normalised ratio; U\&Es, urea and electrolytes; XRWH, Royal Wolverhampton Hospital.

pathway in a timely manner (figure 1 ). In addition to streamlining referral pathways, both groups were keen to empower GPs to undertake more of the diagnosis, management and follow-up of GI conditions. Evidence suggests that with adequate support, chronic disease management can be both safe and effective in primary care. ${ }^{3}$

A blinded retrospective audit of 300 GI referrals was undertaken to ensure clinical care and patient safety would not be compromised and to determine potential efficiency savings which would inform appropriate financial modelling. This supported our proposals and allowed formal introduction of CAS in 2014.

We now present data from the first 3 years of the GI CAS triage system at RWT and assess its financial and operational impact.

\section{METHODS}

\section{Service development}

The development of this service was agreed with Wolverhampton CCG, whose support was integral to the process. Eighteen surrounding CCGs also agreed to adopt the process for GI referrals to RWT.
Following analysis of the pilot study, we anticipated that two new OP clinic sessions could be switched to CAS referral triage sessions. That would also allow sufficient additional time for managing the administrative work resulting from the process. Given that these sessions were flexible, there was the opportunity to undertake them at any point during the week, thus providing greater flexibility for the consultant workforce. As the number of referrals increased, the allocated time required was also reviewed. We identified that minimising the number of consultants undertaking triage (to 2 ) greatly improved the consistency of the process.

CAS was introduced to primary care physicians and patients by the CCG in January 2014 as the main pathway for GI referrals to RWT. Virtual CAS clinics were available for GPs to book patients directly although conventional referral methods remained opened until familiarity with the system was achieved. Written information was developed for both referring GPs and patients. The 2 -week wait referral system was not altered and ran alongside CAS. Consultant to consultant referrals are seen by the consultant to whom the patient is 

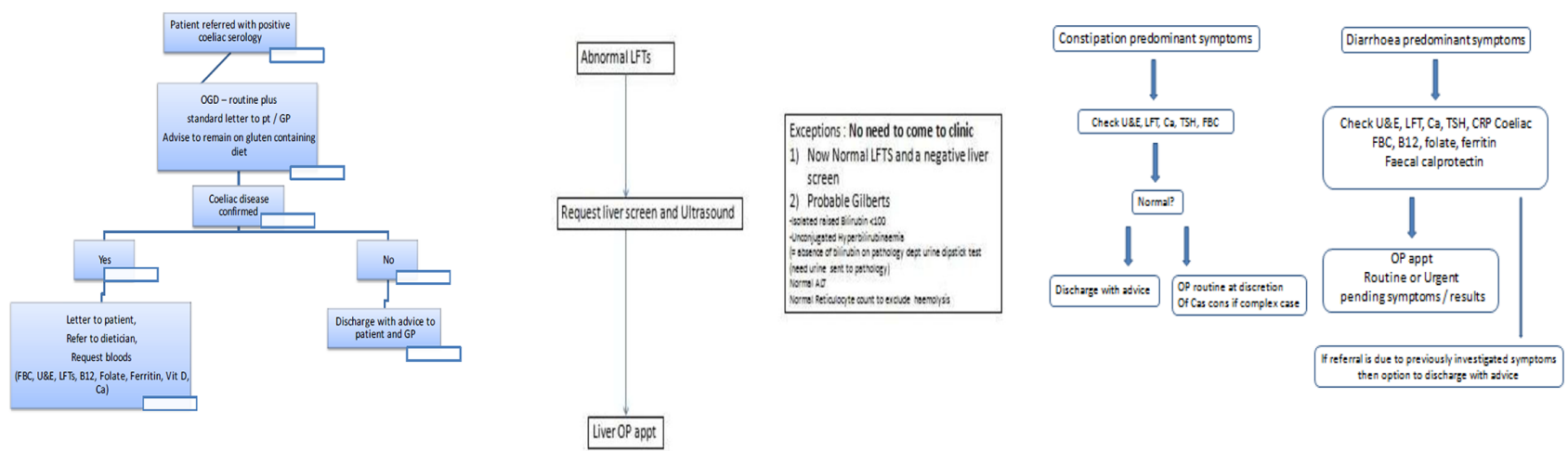

CAS rectal bleeding pathway

\section{CAS dyspepsia pathway}
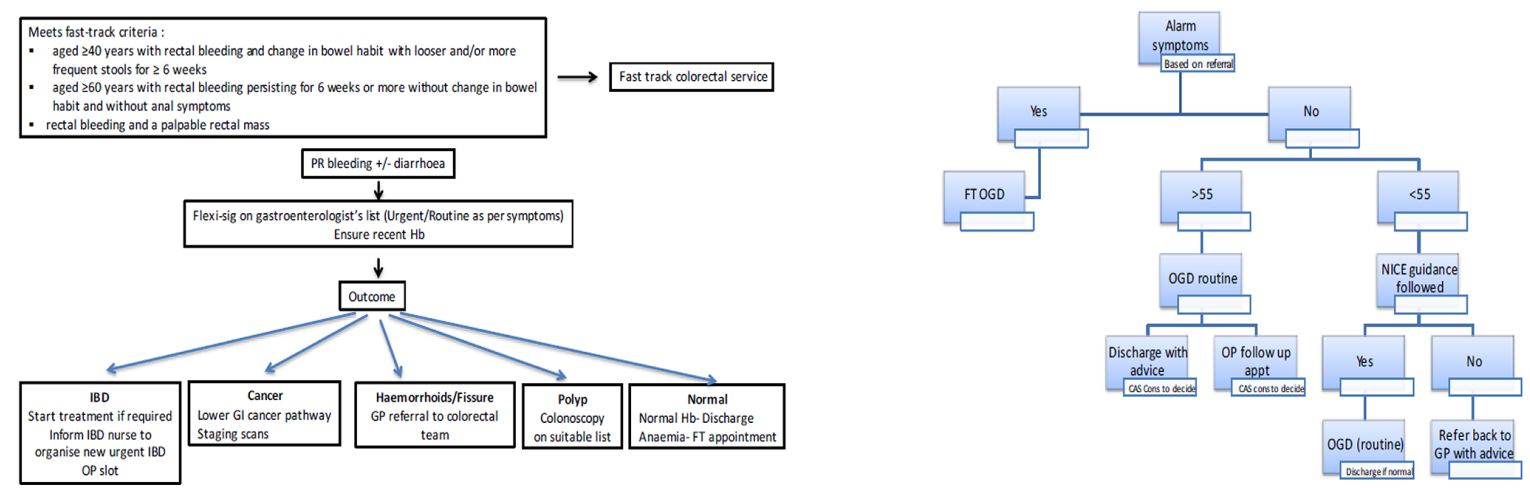

Figure 2 CAS pathways. CA, cancer; CAS, Clinical Assessment Service; FBC, full blood count; FT, fast track; Hb, haemoglobin; NICE, National Institute for Clinical Excellence; TSH thyroid-stimulating hormone.

referred to, and they have the option of directing the referral to CAS or opt to make individual arrangements to review these patients. All non-fast track referrals are now processed through CAS.

Information technology development and proforma

An electronic CAS proforma and data platform were developed by the IT department at RWT to allow electronic triage of patients and also to provide anonymised outcome data for monitoring and auditing purposes. The CAS proforma includes patient demographics, indication for referral, investigations required and patient outcome decision (figure 1).

\section{Outcomes and pathways}

Five potential outcomes are identified for each patient: direct to new OP appointment in the GI clinic, investigations prior to a new OP appointment, direct referral to diagnostic tests (endoscopy or radiology), return to GP for primary care management with specialist advice or inappropriate for the specialty and return to the GP with relevant advice or onward internal referral to appropriate specialty. Nominated GI consultants were given allocated sessions to review and action referrals with an agreed timeframe of 3 weeks from GP referral being received at RWT to the patient being aware of the management plan.

Pathways for the five most common referral indications (dyspepsia, coeliac disease, rectal bleeding, change in bowel habit and deranged liver function tests) were developed with primary care to ensure consistent and optimal patient triage (figure 2). These pathways incorporate national guidance and offer the ability to empower GPs to manage common GI conditions in primary care and to provide a standard for internal audit of practice.

\section{Administration}

To manage the demand of extra administrative work generated from the CAS, a dedicated full-time administrator was appointed. This role includes processing of triaged e-proformas and communicating directly with GPs and patients to facilitate efficient running of the service. In order to minimise administrative tasks, a series of standard letters with clear information were developed for each outcome.

\section{Data collection and analysis}

An electronic CAS database was used to collect and retrospectively analyse data from January 2014 to December 2016. 

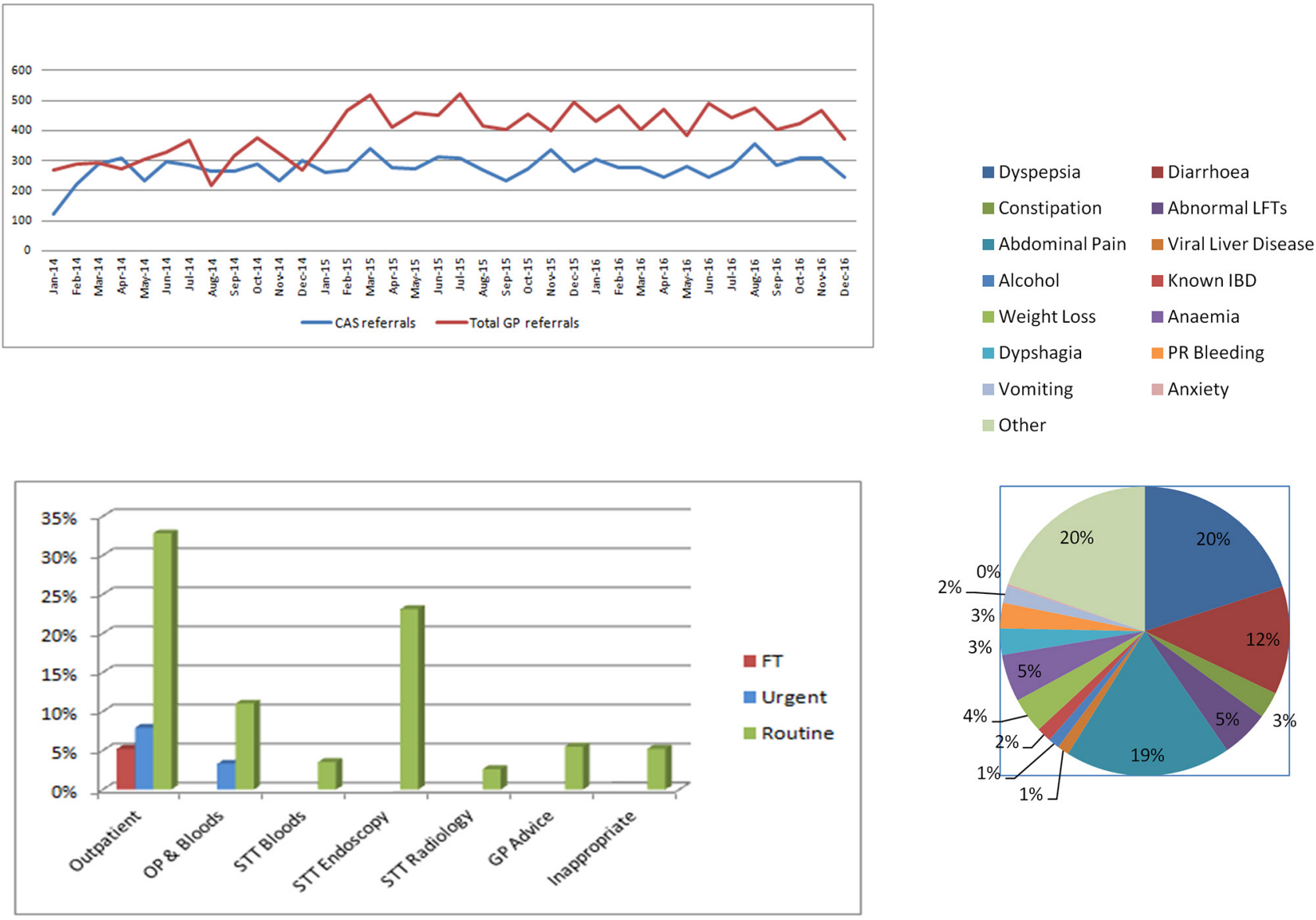

Figure 3 Monthly distribution of total and CAS clinic referrals during 2014-2016. CAS outcomes during 2014-2015. CAS referrals indications. CAS, Clinical Assessment Service; STT, straight to test.

Reports on departmental performance were obtained from our information analysts group. A financial model was used to calculate the economic impact of our services.

\section{RESULTS}

A total of 14245 GP referrals were received during the delivery of the new service between January 2014 and December 2016 (36 months). The mean monthly total referrals were $395( \pm 80)$ (figure 3 ). A total of 9773 referrals were triaged via our CAS. The stepwise increase in demand in December 2014 was due to RWT integrating with the Cannock and Surrounds population (size: 140000) following the dissolution of the Mid Staffordshire NHS Trust. Owing to amalgamation of trusts, there was a period of time when some patients from the CCGs attached to Mid Staffordshire Trust were referred through choose and book, which has now changed.

\section{Indications}

The main indications for referral included dyspepsia, abdominal pain and diarrhoea. A detailed distribution is shown in figure 4 . Some patients had more than one indication for referral.

\section{CAS outcomes}

A new OP appointment was offered to $60.1 \%$ $(\mathrm{n}=5873)$ either as a fast track, urgent or routine slot. A total of $23.8 \%(n=2326)$ of these patients had investigations arranged prior to their OP appointment (eg, blood tests, faecal calprotectin and Helicobacter pylori testing).

Endoscopic or radiological investigations were requested for 29.2\% $(n=2854)$ of patients prior to deciding on further management plan. Out of those, $26.8 \%(n=765)$ went on to receive another GI clinic appointment. This meant that the remaining patients were discharged back to their GP following one of the above investigations which were corresponding to $21.3 \%(n=2089)$ of all CAS patients.

A total of $5.5 \%(n=538)$ were discharged back to primary care with a letter of advice.

A total of $5.2 \%(n=509)$ were deemed inappropriate for GI clinic and were redirected to other specialists.

Overall, 32\% $(n=3136)$ of patients were managed without a face to face appointment in the GI clinic. For the 3-year period of delivering the service, this corresponds to 3136 less OP appointments which equate to 448 new OP clinics (as defined by British Society of Gastroenterology (BSG) guidance). 


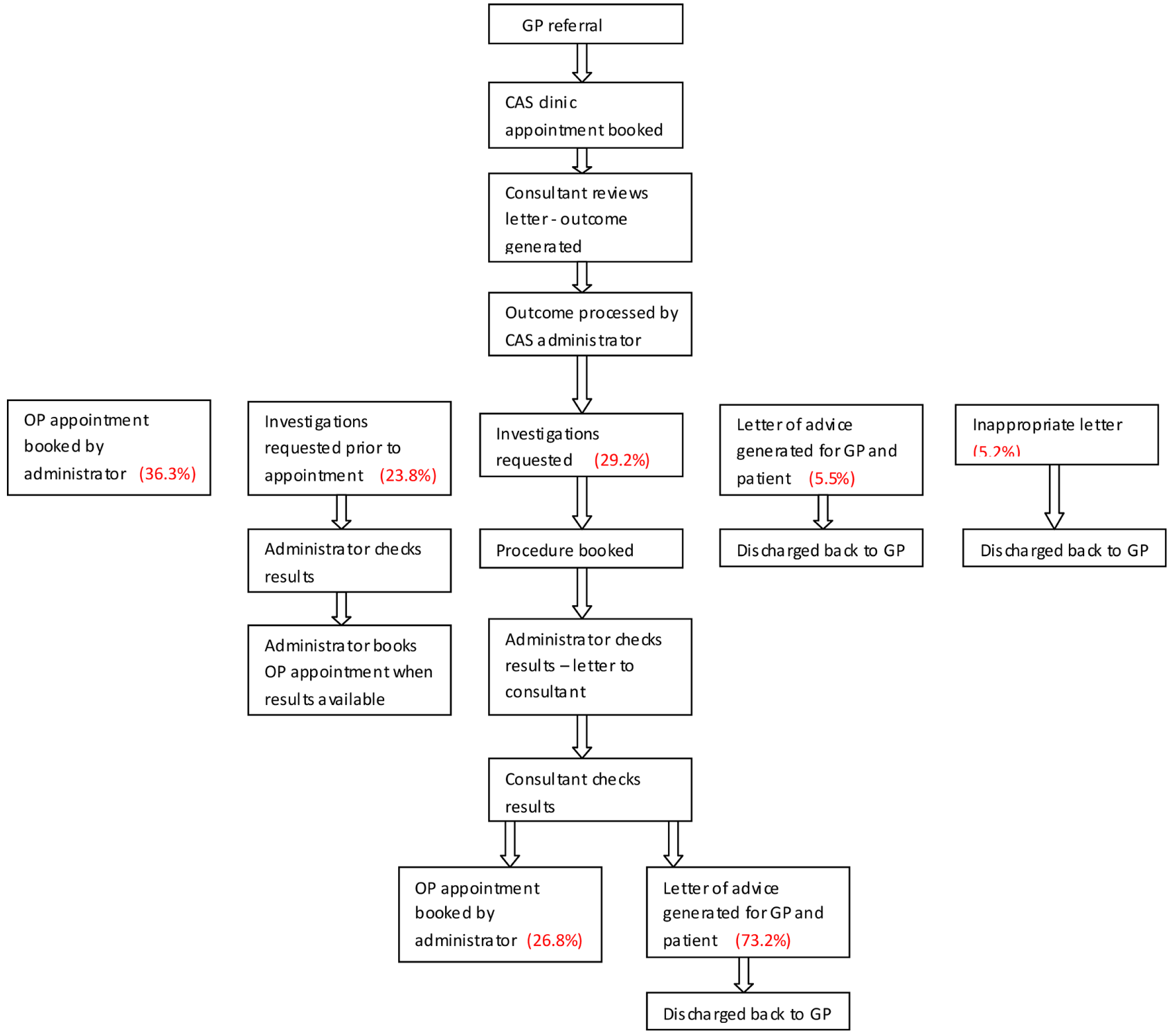

Figure 4 Annotated CAS flowchart. CAS, Clinical Assessment Service.

Based on current CAS capacity (112 slots per week), 87 CAS sessions were required for review of all referrals received $(n=9773)$. Therefore, in total, 361 clinical sessions were saved.

A detailed annual analysis of the various outcomes of CAS triage is presented in figures 3 and 4.

\section{Performance indicators}

Following the introduction of CAS, the departmental 18 -week performance increased from $96.5 \%$ to $98.5 \%$ in 2014 . Despite increasing our population by $140 \mathrm{~K}$ with the integration of Cannock \& Surrounds CCG in 2015, the 18-week performance remained stable during the following 2 years at $96.4 \%$ and 95.8\% accordingly.

A similar trend was observed in the waiting times for a new OP appointment. During 2014, the average wait for an appointment reduced from 53.8 to 32.2 days. This increased over the next 2 years to 47.2 in 2015 and 51.4 days in 2016. These figures are, however, well within the 12-week target despite a significant overnight increase in demand. It should be also noted that the above waiting times include a number of hospital contacts/tests for many patients before their attendance to OP.

The capacity of CAS clinics has been steadily expanding over the 3 -year period in order to accommodate higher referral demand and maintain a 3-week wait window for initial triage. We currently triage 112 OP referrals per week after recent increase in capacity in order to match the demand for all GP referrals to ensure that there is a consistent pathway for all patients.

\section{DNA and re-referral rates}

The DNA rate for all new referrals prior to implementation of CAS was 14\%. This dropped to 7\% in 2015 and to $8.52 \%$ in 2016 . 
Re-referral to GI CAS was monitored to determine if patients who did not receive a face to face appointment were simply being re-referred, and if any significant pathology had been missed by initial triage. The total re-referral rate for patients not seen in an OP clinic following the first referral was $0.5 \%$. Re-referral was considered the acceptance of a second CAS referral with the same presenting complaint within 12 months. In each of these cases, patients were offered a face to face consultation. No serious pathology (cancer, inflammatory bowel disease, peptic ulcer disease and chronic liver disease) was missed in this cohort of patients.

\section{Financial impact}

The financial benefits realised owing to reduced face to face attendance at OP clinics can be determined by modelling. Using the percentage of patients not requiring an OP appointment, it is feasible to calculate gross savings for the health economy. This is offset by the agreed cost of CAS referrals. Consultant time to triage referrals was made available by the reduction in the number of face to face clinics required and therefore has no direct cost.

Over the 3-year period, CAS resulted in an estimated reduced expenditure by the Health Economy of $£ 481613$. The formula used was [(Outpatient clinic tariff) - (CAS clinic tariff) $\times$ Number of outpatients avoided].

If the service had been able to offer capacity throughout for all GP referrals, it could have saved approximately $£ 701993$. It should be noted that although CAS has resulted in 32\% of patients not requiring an OP appointment, demand for the GI service has continued to rise so it has not resulted in less patients coming to clinic overall. Therefore, without this service, the OP clinics for gastroenterology would have increased.

It would be feasible to do further economic modelling to demonstrate the potential cost benefits; however, this is beyond the scope and purpose of this paper.

\section{Feedback}

To evaluate GPs' opinion about CAS, a survey was conducted in 2014, after the first year of implementation. Despite a disappointing response rate of $10 \%$, comments were positive with $80 \%$ reporting that CAS approach was acceptable and the service easy to use, while all felt that advice provided by the consultant was comprehensive.

To assess patients' satisfaction, questionnaires were offered to those who attended the clinic. Number of responses again was low (11 patients) but with $90 \%$ of them being very satisfied with the information and the quality of care they received.

\section{DISCUSSION}

Developing and introducing a new service is challenging as it requires collaboration of various but equally important groups in different organisations. In the health sector, risks and benefits need to be carefully balanced for a cost-effective but patient-centred service.

Virtual clinics or use of guidelines for referral have been developed as an alternative way to cope with increased pressure with good outcomes. ${ }^{45}$ The information given in referral letters is often sufficient to determine what investigations patients are likely to require or whether they are more suitable for an alternative management pathway. However, in traditional Choose and Book (C\&B) systems, it can be difficult and time consuming to try and alter the patient's flow or arrange tests prior to an OP appointment. CAS triage was created in order to give the specialist more control on the patient pathway and deliver a more efficient service.

The introduction of the CAS has provided several clinical benefits. Patients whose symptoms require an OP appointment are seen within shorter times. This is likely to be a factor in the reduced DNA rate also observed. Similarly, patients suitable for direct to test pathway are being provided with a diagnosis, treatment or reassurance in a more efficient manner. If ongoing concern, an OP appointment can be requested electronically. All data is captured on our database and allows internal monitoring and identification of variance in practice between consultants.

The ability to provide bespoke feedback and advice to patients and GPs for those patients who were deemed not to require face to face appointments has also proved beneficial. Where appropriate (eg, coeliac disease, irritable bowel syndrome and dyspepsia), our standard letters contain links to patient organisations and regulated online information which empowers patients to self-manage their conditions. Additionally, those inappropriate for the specialty referrals are fed back to the CCG who is then able to identify potential educational needs in primary care and coordinate appropriate interventions.

Besides the clinical benefits, we have achieved significant financial savings to the health economy. Our results over 3 years have demonstrated that on average, $32 \%$ of patients referred do not require a face to face consultation resulting in gross savings of $£ 481169$. While there are administrative costs to be considered, it is clear that the CAS offers real value for money. There has been considerable focus on referral management schemes recently suggesting that these schemes are expensive to commission and provide uncertain financial and clinical returns. ${ }^{6}$ There are several key differences in our CAS which is underpinned by close collaborative work between the CCG and RWT to ensure patient safety and good clinical care, and through this, we have been able to demonstrate not only improved efficiency but also significantly reduced cost of GI OP services to the local commissioners.

The concern of missing a serious diagnosis in the proportion of patients not seen in a face to face clinic was addressed by rigorous monitoring and a robust governance agreement with primary care. Best practice 
guidance for using CAS functionality within choose and book was agreed; therefore, once a booking had been made into CAS and the referral letter has been attached, the clinical responsibility was transferred to the CAS provider. ${ }^{7}$ Clinicians also agreed that if the triage concludes with the patient being returned for management within primary care then, once the referral is returned with advice, the responsibility for the patient returns to the GP.

To date, we are not aware of any clinical significant diagnoses that have been missed or compromised by the introduction of the CAS. Moreover, GPs are granted the ability to override the decision taken if they felt that the triage decision was not appropriate or if they had significant concerns which were not addressed. Discussions held in a patient focus group positively acknowledged the direct to test approach with need for less hospital visits and the appropriate

\section{Key messages}

\section{What is already known on the topic?}

- Increasing demand for specialist referrals from primary to secondary care puts pressure in NHS resources.

- Number of referral management schemes has been developed by CCGs but with uncertain financial and clinical benefits.

\section{What this study adds?}

- This study is the first to evaluate the impact of a new referral triage system.

- With appropriate services in place and close collaboration with the CCG, there could be a significant reduction in need for face to face consultations without compromising patients' care, associated with considerable reduced expenditure by the health economy.

How might it impact on clinical practice in the foreseeable future?

- Our 'Clinical Assessment Service' model has the potential to be adapted by other specialties and organisations with reproducible results. specialty redirection for those that would not benefit from specialist GI advice.

The model of clinical assessment we have developed for GI referrals has the potential to be adopted by other specialties and organisations. Indeed within RWT, the renal directorate adopted the CAS system. This would certainly also be feasible for other organisations but would require local adaptations to suit available systems and resources. We believe the success of this process is a combination of patients' cooperation along with the collaborative interactions between the clinical and financial teams from both the CCG and RWT.

Acknowledgements We acknowledge Jackie Mason, CAS coordinator, of this study.

Contributors SP conducted and submitted the study. CH, DG, AS and DB planned the study. SM planned and conducted the study. BM planned, conducted and submitted the study.

Competing interests None declared.

Provenance and peer review Not commissioned; externally peer reviewed.

(C) Article author(s) (or their employer(s) unless otherwise stated in the text of the article) 2018. All rights reserved. No commercial use is permitted unless otherwise expressly granted.

\section{REFERENCES}

1 Khong TL, Naik K, Sivakumar R, et al. Impact of the United Kingdom national bowel cancer awareness campaigns 2012 on colorectal cancer services and patient survival. Colorectal Dis 2015;17:1055-61.

2 Barnett ML, Song Z, Landon BE. Trends in physician referrals in the United States, 1999-2009. Arch Intern Med 2012;172:163-70.

3 Winpenny E, Miani C, Pitchforth E, et al. Outpatient services and primary care: scoping review, substudies and international comparisons. Health Services and Delivery Research 2016;4:1-290.

4 Mariotti G, Gentilini M, Dapor V. Improving referral activity on primary-secondary care interface using an electronic decision support system. Int J Med Inform 2013;82:1144-51.

5 Ho CK, Boscardin CK, Gleason N, et al. Optimizing the prereferral workup for gastroenterology and hepatology specialty care: consensus using the Delphi method. J Eval Clin Pract 2016;22:46-52.

6 Iacobucci G. Referral management schemes: good for whom? BMJ 2017;356:i6856.

7 Department of Health. Responsibilities and operational requirements for the correct use of choose and book, 2009. www.mysurgerywebsite.co.uk/website/IGP367/files/CB\%20-\% 20responsibilities\%20and\%20requirements.pdf (accessed 9 Feb 2017). 